\title{
CARACTERIZAÇÃO FÍSICO-QUÍMICA DE FRUTOS DE CLONES DE DOVIÁLIS (Dovyalis abyssinica Warb) ${ }^{1}$
}

\author{
JOSÉ ANTONIO ALBERTO DA SILVA², REGINA KITAGAWA GRIZOTTO², \\ FERNANDO BERGANTINI MIGUEL ${ }^{2}$, IVANA MARINO BÁRBARO ${ }^{2}$
}

RESUMO - A Doviális é uma fruta exótica originária da África, de coloração laranja-avermelhada e elevada acidez. No Brasil, foi propagada via sementes na Estação Experimental de Citricultura de Bebedouro-SP, pela FCAV/UNESP a partir de uma planta introduzida da Flórida-USA, sendo selecionada uma planta por apresentar frutos com menor acidez. Esta planta, denominada doviális 'Romana', encontra-se em plena produção e vem sendo propagada vegetativamente. O objetivo deste trabalho foi avaliar o potencial de utilização dos frutos de dovialis para o mercado de fruta fresca ou industrial, considerando as características físicas e físico-químicas dos frutos. Este estudo preliminar é inédito, pois há poucos estudos de aproveitamento dos frutos de doviális na literatura. Os resultados mostraram que a acidez dos frutos da doviális 'Romana' ( $1,76 \%$ de ácido cítrico) foi significativamente inferior aos da planta introduzida (5,5\% de ácido cítrico). Os frutos da doviális 'Romana' também apresentaram 'ratio' elevado $(7,55)$ e coloração da polpa tendendo para o amarelo-esverdeado $\left(a^{*}=9,01, b^{*}=33,15\right)$, significativamente diferentes da polpa da planta introduzida. Em geral, não houve diferenças significativas em rendimento em polpa (79\%), sólidos solúveis (12 ${ }^{\circ}$ Brix $)$, diâmetros transversal $(26 \mathrm{~mm})$ e longitudinal $(23 \mathrm{~mm})$. Desta forma, podemos sugerir que os frutos da doviális 'Romana' apresentam aptidão tanto para o mercado ao natural como para a produção de doces e sucos. Já os frutos da planta introduzida, face à elevada acidez e coloração atrativa, destinam-se à produção de doces e sucos. $\mathrm{O}$ aspecto visual da fruta e o sabor característico da doviális 'Romana' abrem potenciais mercados para a diversificação da produção comercial na fruticultura exótica.

Termos para indexação: fruta exótica, diversificação, fruticultura.

\section{PHYSICAL AND CHEMICAL CHARACTERIZATION OF FRUITS OF DOVYALIS CLONES (Dovyalis abyssinica Warb)}

\begin{abstract}
The Dovyalis is an exotic fruit originated in Africa, reddish-orange color and high acidity. In Brazil it was propagated through seeds in Citrus Experimental Station Bebedouro SP, from a plant introduced from Florida-USA, by FCAV/UNESP being selected a plant produced fruits with lower acidity. This plant, called dovyalis 'Romana', is in full production, and has been vegetatively propagated. The objective was to evaluate the potential use of the fruits of dovyalis for the fresh fruit market or industry, considering its physical and physical-chemical characteristics. This preliminary study is unprecedented, because there are few studies use about the uses of the dovyalis in literature. The results showed that the acidity of the fruits of dovyalis 'Romana' ( $1.76 \%$ citric acid) was significantly lower than those of the introduced plants ( $5.5 \%$ citric acid). The fruits of dovyalis 'Romana' also had high ratio (7.55) and the pulp color tends to yellow-green $\left(\mathrm{a}^{*}=9.01, \mathrm{~b}^{*}=33.15\right)$, significantly different from the pulp of the introduced plant. In general, no significant differences in pulp yield (79\%), soluble solids $\left(12{ }^{\circ} \mathrm{Brix}\right)$, transversal diameter $(26 \mathrm{~mm})$ and longitudinal diameter $(23 \mathrm{~mm})$. Thus, we suggest that the fruits of dovyalis Romana present ability to both natural market and production of sweets and juices. However, the fruits of the introduced plant, in concern of the high acidity and the attractive colour, are intended to the production of sweets and juices. The visual appearance of the fruit and flavor of the dovyalis 'Romana' open up potential markets for the diversification of commercial production of exotic fruits.
\end{abstract}

Index terms: exotic fruit, diversification, fruiticulture.

\footnotetext{
${ }^{1}$ Trabalho Sinfruit 212 - Simpósio Internacional de Fruticultura - Avanços na Fruticultura (17 a 21 Outubro)

${ }^{2}$ Pesquisador Científico, Pólo Regional da Alta Mogiana, APTA, Avenida Rui Barbosa s/n. ${ }^{\circ}$, C.P. 35, CEP 14770-000, Colina-SP, Fone (17) 3341-1400. E-mail: jaas@apta.sp.gov.br
} 


\section{INTRODUÇ̃̃O}

Atualmente, com a abertura comercial, o mercado mundial de frutas tem-se mostrado mais competitivo e aberto a novidades, como as frutas nativas e exóticas. As divulgações na mídia sobre os benefícios do consumo de frutas, destacando-as como alimento saudável, balanceado, funcional e diversificado, com suas cores, formatos, cheiros e sabores aguçam o desejo dos consumidores por frutas nativas e exóticas, demandando crescimento e qualificação da cadeia de produção, onde se insere a pesquisa científica.

Anteriormente consideradas como nicho de mercado, algumas espécies exóticas já caíram no gosto dos consumidores que aguardam a época de produção para se deliciarem com as lichias, uvas, kiwis, pitayas, dentre outras, sendo produzida no País como diversificação econômica da fruticultura. Porém, não foram encontrados números e valores oficiais da produção e comercialização destas frutas.

A doviális (Dovyalis abyssinica Warb. x $D$. hebecarpa Warb.) é uma espécie da família Salicaceae (Flacourtiaceae), originária da África, também conhecida como mukambura, unkokolo, groselha-do-ceilão, abricó-da-flórida, groselha-híbrida, keyapple, dove plum ou ketembilia. O fruto de doviális tem formato esférico, com polpa suculenta e naturalmente ácida, característica desejada para a fabricação de doces, geleias e sucos. Possui uma fina pele com coloração de laranja a vermelho-arroxeada, é recoberta por uma macia camada de pelos que proporciona uma textura bastante agradável ao toque. As sementes pequenas e facilmente removíveis resultam em alto rendimento em polpa e confirmam sua aptidão para o processamento. Os frutos, quando colhidos, mantêm o cálice aderido ao fruto, dando aspecto de frescor e realçando o aspecto de uma fruta diferente das produzidas comercialmente.

A doviális é uma espécie com bom potencial de mercado que poderá trilhar o caminho das frutas exóticas com plantios comerciais no Brasil. Introduzida na Flórida, chegou ao Brasil através de um programa de introdução de espécies coordenado pelo professor Dr. Luiz Carlos Donadio, da UNESP de Jaboticabal, e daí distribuído para a Estação Experimental de Citricultura de Bebedouro, localizada em Bebedouro-SP, e para o Polo Regional de Desenvolvimento Tecnológico dos Agronegócios da Alta Mogiana (PRDTA-Alta Mogiana), órgão da Secretaria de Agricultura, localizada em Colina-SP.

Originalmente, os frutos da doviális são extremamente ácidos, não servindo para o consumo ao natural, apesar de o fruto apresentar formato e cor bastante atrativos. Por ocasião da propagação de mudas via sementes, na Estação Experimental de Bebedouro, foi possível selecionar uma planta em que os frutos apresentaram menor acidez. Supôs-se, desta forma, ser possível aliar dupla aptidão ao fruto da doviális, podendo atender aos mercados de frutos para a indústria e consumo ao natural. A planta selecionada que produz frutos de menor acidez, foi nomeada de variedade Romana e vem sendo propagada vegetativamente via alporquia e estaquia, conforme recomenda Donadio et al. (1998), sendo este um dos primeiros estudos tecnológicos de qualificação.

O objetivo geral deste trabalho foi avaliar comparativamente os frutos de duas plantas distintas de doviális (Dovyalis abyssinica Warb.), cultivadas na Estação Experimental de Citriculutra de Bebedouro, por meio de análises físicas e físico-químicas. $\mathrm{O}$ objetivo específico foi estabelecer o potencial dos frutos para os mercados ao natural e industrial.

\section{MATERIAL E MÉTODOS}

As plantas de doviális com frutos considerados de maior e menor acidez estão plantadas na Estação Experimental de Citricultura de Bebedouro (EECB), na região norte do Estado de São Paulo, latitude $20^{\circ} 53^{\prime} 16^{\prime \prime} \mathrm{S}$, longitude $48^{\circ} 28^{\prime} 11^{\prime \prime} \mathrm{W}$ e altitude de $601 \mathrm{~m}$.

O plantio foi realizado em 2002, a partir de mudas oriundas de sementes. Após dois anos em plantio sem irrigação, houve a frutificação, sendo possível identificar, entre várias, uma planta com menor acidez nos frutos, nomeada informalmente de doviális "Romana". As mudas produzidas a partir desta planta-matriz pelo processo de alporquia e estaquia mantiveram as características originais com boa produção de frutos doces.

As características dos frutos de doviális foram avaliadas em amostras de aproximadamente dois quilos de frutos de duas plantas consideradas de maior e menor acidez, na fase de maduros, em junho de 2011, e enviados ao laboratório de análises do PRDTA-Alta Mogiana, unidade da APTA - Colina-SP.

Para facilitar a diferenciação entre as plantas, os frutos de maior e menor acidez foram denominados de doviális e doviális Romana, respectivamente. Os frutos foram armazenados sob refrigeração $\left(9{ }^{\circ} \mathrm{C}\right)$ e analisados no dia seguinte, conforme descritos a seguir:

1) Características Físicas dos Frutos: medida dos diâmetros polar e equatorial $(\mathrm{mm})$, com auxílio de paquímetro digital, e massa dos frutos (g), com balança digital; 
2) Rendimento em Suco (RS): calculado em porcentagem da massa do suco da amostra, em relação à massa do fruto, conforme Equação 1:

$R S(\%)=\frac{\text { massa suco }}{\text { massa fruto }} x 100$

3) Resíduo: calculado em porcentagem da massa de resíduo em relação à massa do fruto, conforme Equação 2:

Resíduo $(\%)=\frac{\text { massa residuo }}{\text { massa fruto }} x 100$

4) Sólidos Solúveis (SS): determinados por refratometria a $20{ }^{\circ} \mathrm{C}$ e expressos em ${ }^{\circ}$ Brix (g $\left.100 \mathrm{~mL}-1\right)$, segundo métodos descrito por Redd et al. (1986);

5) Acidez Titulável (AT): determinada por titulometria com solução de hidróxido $0,3125 \mathrm{~N}$ até $\mathrm{pH} 8,1$, e os resultados expressos em gramas de ácido cítrico por $100 \mathrm{~mL}$ de suco, de acordo com os métodos analíticos citados por Redd et al. (1986), registrando o $\mathrm{pH}$ inicial do suco, com potenciômetro modelo DM 22, marca Digimed;

6) Índice de Maturação ou "Ratio": obtido por cálculo, dividindo-se o teor de sólidos solúveis (SS) pela acidez titulável (AT);

7) Cor Objetiva: a cor foi avaliada em colorímetro modelo CR 300, marca Minolta. O sistema de leitura utilizado foi o CIELAB, representado pelos seguintes parâmetros: coordenada $L^{*}$ (luminosidade), coordenada de cromaticidade $\mathrm{a}^{*}(-\mathrm{a}=\mathrm{verde}$, $+\mathrm{a}=$ vermelho) e a coordenada de cromaticidade $\mathrm{b}^{*}$ $(-\mathrm{b}=\mathrm{azul},+\mathrm{b}=$ amarelo $)$. As amostras foram colocadas em placa de Petri, a $3 \mathrm{~mm}$ de caminho óptico;

8) Matéria Seca: determinada em estufa com circulação de ar modelo TE 393/1, marca Tecnal, a 55 ${ }^{\circ} \mathrm{C}$ até peso constante. $\mathrm{O}$ tempo total foi de aproximadamente $120 \mathrm{~h}$ (5 dias), em função da baixa temperatura de secagem;

8) Proteína Bruta: determinada conforme metodologia de micro Kjeldhal (AOAC, 2005);

9) Extrato Etéreo: determinado em extrator de gordura tipo Soxhlet (AOAC, 2005);

10) Cinzas: determinadas em forno mufla a $550{ }^{\circ} \mathrm{C}$ (AOAC, 2005).

As análises de proteína bruta, cinzas e extrato etéreo foram realizadas em duplicatas, por motivo de limitação de material. As demais análises foram realizadas com seis ou mais repetições, e os valores obtidos foram avaliados com o auxílio do programa Statistica versão 5.0 para comparação entre as médias, pelo teste de Tukey $(\mathrm{p} \leq 0,05)$.

\section{RESULTADOS E DISCUSSÃO}

Na Tabela 1, são apresentados os resultados das análises físico-químicas e do rendimento em suco realizado nos frutos de doviális provenientes de duas plantas com características distintas.

Constatou-se que o rendimento em polpa (79\%) e os parâmetros de desenvolvimento do fruto (diâmetros transversal e longitudinal) foram iguais nos frutos de doviális ácida e menos ácida. Porém, as sementes de doviális 'Romana' eram ligeiramente maiores, tanto em tamanho como em número por fruto, resultando no maior percentual de resíduo $(17,5 \%)$.

Analisando frutos de doviális, Cavalcanti e Martins (2005) obtiveram rendimento médio de polpa de $75 \%$, considerando esta uma boa qualidade física para o mercado. Donadio et al. (2002), avaliando frutas nativas da flora brasileira, citam o rendimento de polpa de $66 \%$ para a pitanga, $69 \%$ para a jabuticaba e $74 \%$ para a cereja-do-rio-grande.

A principal diferença dos frutos de doviális 'Romana' está na menor acidez $(1,76 \%$ de ácido cítrico) e, consequentemente, maior Índice de Maturação ou 'ratio' $(7,55)$, em comparação aos frutos de doviális da planta introduzida, cujos valores de acidez e 'ratio' foram 5,5\% de ácido cítrico e 2,16, respectivamente. O camu-camu, fruta nativa da região Amazônica, apresentou acidez elevada, 2,78 \% de ácido cítrico, em comparação à pitanga $(1,8 \%$ de ácido cítrico) e uvaia (1,53\% de ácido cítrico), conforme Donadio et al. (2002).

$\mathrm{O}$ pH de doviális e de doviális 'Romana' ficou em torno de 3,0, não diferiu significativamente $(\mathrm{p} \leq 0,05)$ e encontra-se próximo dos valores de pH encontrados para acerola $(2,8)$, cajá $(2,9)$, murici $(3,7)$ e tamarindo $(2,5)$, conforme Canuto et al.(2010).

O 'ratio', expresso pela relação Sólidos Solúveis (SS) e Acidez Titulável (AT), indica o grau de equilíbrio entre o teor de açúcares e os ácidos orgânicos do fruto (VIÉGAS, 1991), estando diretamente relacionado à qualidade quanto ao atributo sabor, sendo, portanto, um importante parâmetro a ser considerado na seleção da variedade do fruto. Segundo Carvalho (2004), essa relação correspondente aos conteúdos de açúcares e de acidez dos frutos é um parâmetro apropriado para medir a percepção de sabor pelo consumidor.

Os frutos de doviális apresentaram 'ratio' baixo, cerca de 2,61, indicando baixo Índice de Maturação. Já a doviális 'Romana' apresentou 'ratio' igual a 7,55 , um pouco abaixo do 9,25 verificado 
em pitanga por Lopes et al. (2005). Em ambos os casos, o baixo 'ratio' é resultante da acidez elevada, 5,5 $\%$ de ácido cítrico para doviális e 1,76 \% de ácido cítrico para a variedade Romana, uma vez que o teor de sólidos solúveis ficou em torno de $12{ }^{\circ}$ Brix. Para efeito de comparação, a laranja-Valência própria para consumo deve apresentar os teores de 1,05\% de ácido cítrico, $11,8^{\circ}$ Brix, e 'ratio' igual a 11, de acordo com Figueiredo (1991) e Pio et al. (2005).

Canuto et al. (2010) encontraram valores de 'ratio' para frutos de acerola, cajá e murici na faixa de 1,$8 ; 4,6$ e 1,5, respectivamente, valores considerados baixos devido aos baixos valores de sólidos solúveis; porém, quando analisou frutos de caju, obteve 'ratio' de 25,0, resultante da razão entre 5,0 ${ }^{\circ}$ Brix de sólidos solúveis e $0,2 \%$ de ácido cítrico de acidez titulável, que são considerados baixos.

O teor de sólidos solúveis em torno de 12 ${ }^{\circ}$ Brix está acima do estabelecido pela legislação Brasileira (BRASIL, 2000), cujo limite mínimo é de 7 g. 100 g-1 de sólidos totais na polpa de fruta. Esse limite estabelece um padrão de qualidade para polpas de frutas comercializadas e objetiva evitar a fraude por diluição com água. $\mathrm{O}$ teor de sólidos solúveis de doviális está acima dos valores encontrados para a pitanga $\left(8,3\right.$ a $\left.11,6^{\circ} \mathrm{Brix}\right)$, uvaia $(7,5$ ${ }^{\circ}$ Brix) e camu-camu $\left(6,35^{\circ}\right.$ Brix $)$, porém abaixo do valor encontrado no araçá ( $\left.16^{\circ} \mathrm{Brix}\right)$, conforme Donadio et al. (2002).

Outro importante fator de qualidade dos frutos é a cor, conforme apresentado na Tabela 2.

As polpas de doviális e doviális 'Romana' apresentaram valores médios para a luminosidade $\mathrm{L}^{*}$ iguais a 30 e 38 , respectivamente, não diferiram significativamente $(\mathrm{p} \leq 0,05)$, e indicaram coloração ligeiramente escura. Quanto mais $\mathrm{L}^{*}$ se aproximar de 100, mais claro é o tom da cor. As coordenadas de cromaticidade $a^{*}=18,60$ (vermelha) e $b^{*}=20,58$ (amarela) da polpa de doviális indicaram predominância da cor amarela sobre a vermelha, resultando na cor laranja-avermelhada, semelhante à cor da manga Ubá (BENEVIDES et al., 2008). A pitanga, por sua vez, apresenta predominância do vermelho $\left(a^{*}=20,67\right)$ sobre o amarelo $\left(b^{*}=15,62\right)$, de acordo com Lopes et al. (2005), resultando na coloração vermelho-amarelada. Os valores elevados de $a^{*} \mathrm{e}$ $b^{*}$ indicam a prevalência dos carotenoides sobre outros pigmentos presentes nesta fruta.

A polpa dos frutos de doviális 'Romana' apresentou coordenada $\mathrm{a}^{*}=9,01$ (verde) e $\mathrm{b}^{*}=33,15$ (amarelo). Desta forma, a cor desta fruta pode ser definida como amarelo-esverdeada, ligeiramente escura $\left(\mathrm{L}^{*}=38\right)$. Podemos associar às diferenças nos valores de $a^{*}$ e $b^{*}$ as possíveis variações climáticas, nutricionais, do ponto de colheita, entre outras, resultando em diferenças na quantidade de carotenoides acumulados.

Os frutos de doviális podem ser considerados boa fonte de vitamina $\mathrm{C}$, pois apresentaram em média $120,3 \mathrm{mg} / 100 \mathrm{~g}$ de fruto fresco, conforme Cavalcanti e Martins (2005).

$\mathrm{O}$ aspecto visual dos frutos de doviális, analisados no PRDTA-Alta Mogiana, pode ser visto nas Figuras 1 e 2.

Cabe ressaltar que os estudos de avaliação da qualidade de doviális estão em andamento, sendo previstos estudos de avaliação sensorial e teste de produção de geleia.

TABELA 1 - Composição centesimal e rendimento em suco de frutos de duas plantas de doviális. Apta Colina 2011.

\begin{tabular}{|c|c|c|c|c|}
\hline \multirow[b]{2}{*}{ Avaliação } & \multicolumn{2}{|c|}{$\begin{array}{l}\text { Doviális } \\
\text { (ácida) }\end{array}$} & \multicolumn{2}{|c|}{$\begin{array}{l}\text { Doviális "Romana" } \\
\text { (menos ácida) }\end{array}$} \\
\hline & Média \pm D.P. & C.V. (\%) & Média \pm D.P. & C.V. $(\%)$ \\
\hline Rendimento Suco (\%) & $79,96 \pm 2,81 \mathrm{a}$ & 3,51 & $78,66 \pm 1,02 \mathrm{a}$ & 1,30 \\
\hline Resíduo (\%) & $11,37 \pm 1,39 b$ & 1,39 & $17,46 \pm 0,54 \mathrm{a}$ & 3,07 \\
\hline Diâmetro Transversal (mm) & $27,50 \pm 1,97 \mathrm{a}$ & 7,16 & $26,27 \pm 1,44 \mathrm{a}$ & 5,49 \\
\hline Diâmetro Longitudinal (mm) & $22,24 \pm 1,25 \mathrm{a}$ & 5,60 & $23,00 \pm 1,60 \mathrm{a}$ & 6,94 \\
\hline Matéria Seca (\%) & $85,43 \pm 0,09 \mathrm{a}$ & 0,11 & $85,11 \pm 0,15 \mathrm{a}$ & 0,18 \\
\hline Acidez Titulável (\% de ácido cítrico) & $5,55 \pm 0,06 \mathrm{a}$ & 1,07 & $1,76 \pm 0,03 \mathrm{~b}$ & 1,78 \\
\hline $\mathrm{Ph}$ & $2,61 \pm 0,07 \mathrm{a}$ & 2,52 & $3,02 \pm 0,07 \mathrm{a}$ & 2,35 \\
\hline Sólidos Solúveis ( $\left.{ }^{\circ} \mathrm{Brix}\right)$ & $11,97 \pm 0,17 \mathrm{a}$ & 1,45 & $13,26 \pm 0,32 \mathrm{a}$ & 2,45 \\
\hline "Ratio" (SS/AT) & $2,16 \pm 0,04 b$ & 2,01 & $7,55 \pm 0,27 \mathrm{a}$ & 3,56 \\
\hline Proteína Bruta (\% b.s.) & $5,63 \pm 0,33 \mathrm{a}$ & 5,81 & $4,41 \pm 0,38 \mathrm{a}$ & 8,56 \\
\hline Extrato Etéreo (\% b.s.) & $7,17 \pm 0,01 \mathrm{a}$ & 0,20 & $7,10 \pm 0,22 \mathrm{a}$ & 3,16 \\
\hline Cinzas (\% b.s.) & $4,14 \pm 0,59 \mathrm{a}$ & 14,26 & $3,92 \pm 0,48 \mathrm{a}$ & 12,30 \\
\hline
\end{tabular}

Médias seguidas de letras iguais, na mesma linha, não diferem significativamente $(p<0,05)$, pelo teste de Tukey. 
TABELA 2 - Cores objetiva e visual da casca e polpa de frutos de duas plantas de doviális, Apta Colina 2011

\begin{tabular}{lccccc}
\hline & \multicolumn{2}{c}{$\begin{array}{c}\text { Doviális } \\
\text { (ácida) }\end{array}$} & \multicolumn{2}{c}{$\begin{array}{c}\text { Doviális 'Romana' } \\
\text { (menos ácida) }\end{array}$} \\
\cline { 2 - 6 } & & Média \pm D.P. & C.V. (\%) & Média \pm D.P & C.V. (\%) \\
\hline Cor objetiva (casca) & L & $32,42 \pm 3,04 \mathrm{a}$ & 9,37 & $34,92 \pm 3,22 \mathrm{a}$ & 9,21 \\
& $\mathrm{a}^{*}$ & $19,25 \pm 2,83 \mathrm{a}$ & 14,69 & $14,40 \pm 2,49 \mathrm{a}$ & 17,27 \\
& $\mathrm{~b}^{*}$ & $19,96 \pm 4,90 \mathrm{a}$ & 24,57 & $18,66 \pm 5,05 \mathrm{a}$ & 27,03 \\
Cor objetiva (polpa) & $\mathrm{L}$ & $30,29 \pm 1,15 \mathrm{~b}$ & 3,80 & $38,09 \pm 0,86 \mathrm{a}$ & 2,27 \\
& $\mathrm{a}^{*}$ & $18,60 \pm 1,76 \mathrm{a}$ & 9,46 & $9,01 \pm 0,40 \mathrm{~b}$ & 4,41 \\
& $\mathrm{~b}^{*}$ & $20,58 \pm 2,02 \mathrm{~b}$ & 9,82 & $33,15 \pm 0,55 \mathrm{a}$ & 1,67 \\
Cor visual & Casca & Amarelo-avermelhada & \multicolumn{2}{c}{ Amarelo-esverdeada } \\
& Polpa & \multicolumn{2}{c}{ Laranja-avermelhada } & \multicolumn{2}{c}{ Amarela } \\
\hline
\end{tabular}

Médias seguidas de letras iguais, na mesma linha, não diferem significativamente $(p<0,05)$, pelo teste de Tukey.

$\mathrm{L}^{*}=$ Luminosidade

$+\mathrm{a}^{*}=$ vermelho; $-\mathrm{a}^{*}=$ verde, $+\mathrm{b}^{*}=$ amarelo, $-\mathrm{b}^{*}=\mathrm{azul}$.

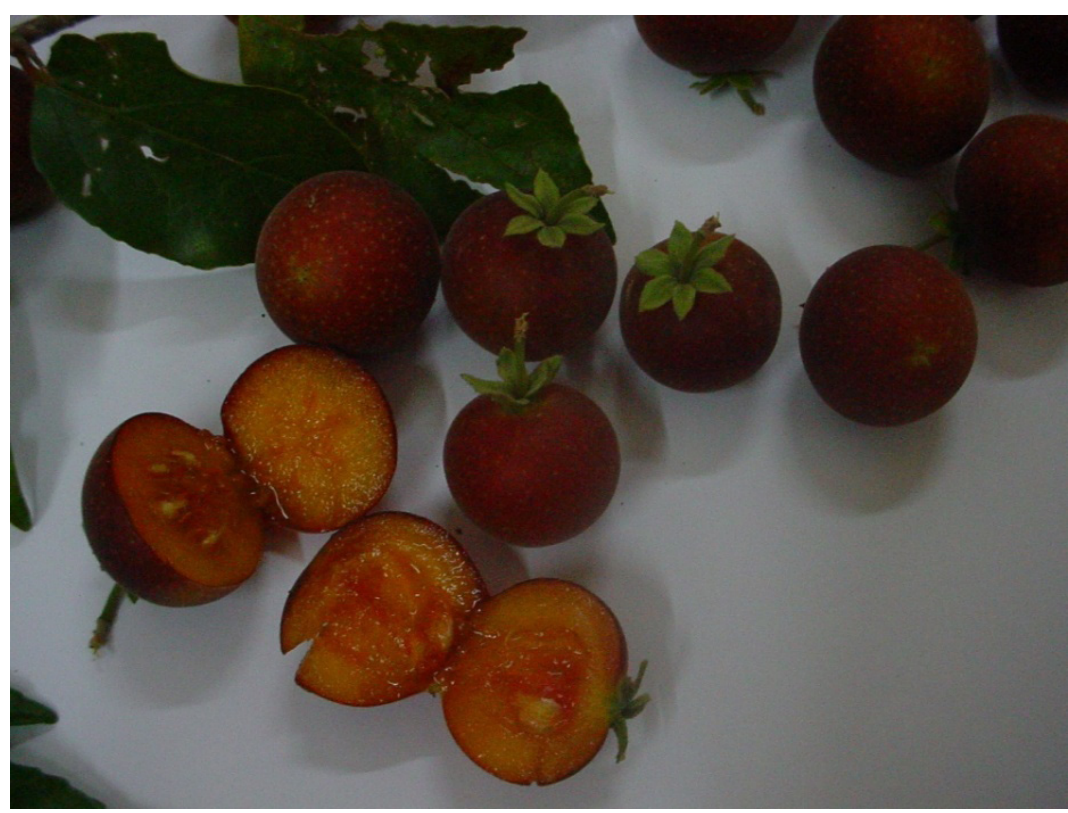

FIGURA 1 - Aspecto visual dos frutos de doviális 'Romana' colhidos na Estação Experimental de Citricultura de Bebedouro. Safra 2011. 


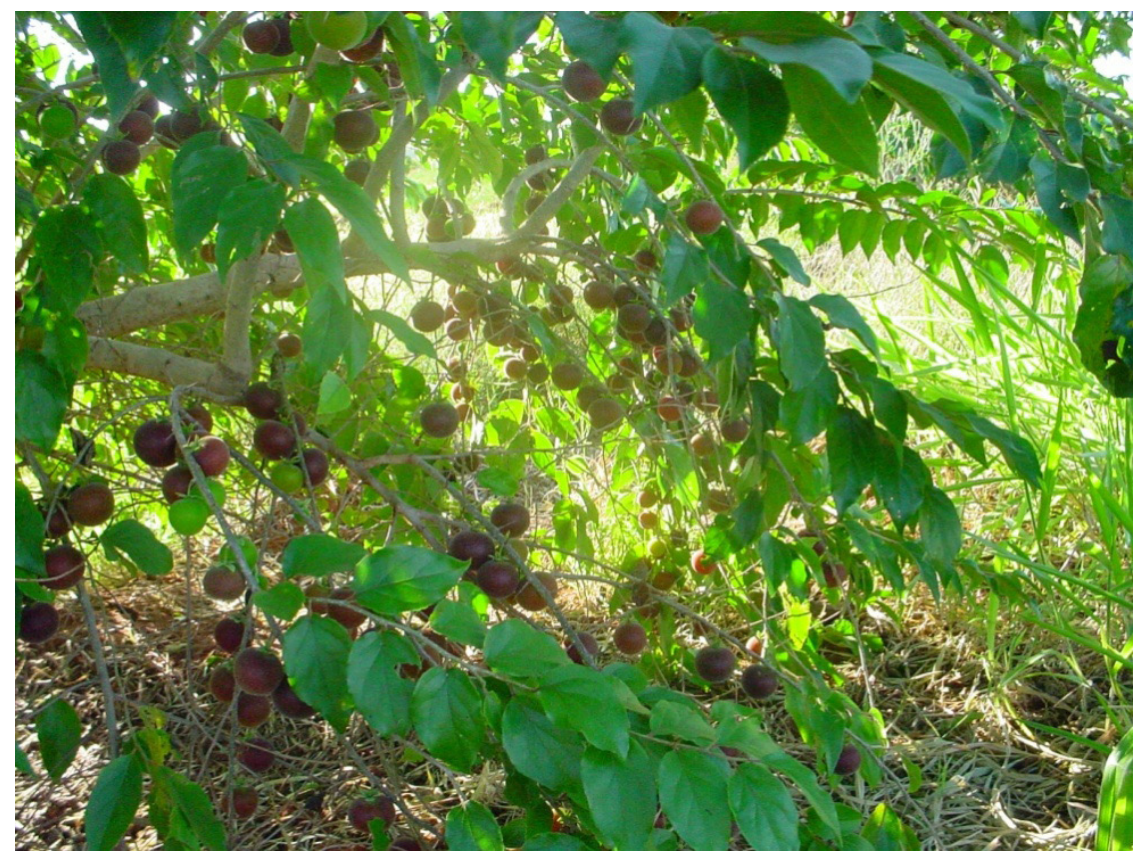

FIGURA 2 - Frutos de doviális 'Romana' na planta introduzida, localizada na Estação Experimental de Citricultura de Bebedouro.

\section{CONCLUSÃO}

1. Podemos concluir que os frutos de doviális destinam-se principalmente à produção de doces e sucos, com coloração da polpa vermelho-amarelada bastante atrativa. Dificilmente, este fruto se adequaria ao consumo ao natural, face à elevada acidez encontrada.

2. A doviális 'Romana' apresenta potencial duplo, ou seja, pode ser utilizada tanto para consumo ao natural como industrial, em função da baixa acidez, significativamente inferior à doviális original. Um ponto desfavorável dos frutos de doviális 'Romana' é a coloração com tendência ao amarelo-esverdeado.

3. As características atrativas tanto visuais como de sabor do dovialis 'Romana', abre um mercado potencial para a diversificação da produção comercial na fruticultura exótica.

\section{AGRADECIMENTO}

Agradecemos à Estação Experimental de Citricultura de Bebedouro-SP, por ceder parte dos frutos para as avaliações.

\section{REFERÊNCIAS}

AOAC. Official methods of analysis of AOAC International. 18. ed. Maryland, 2005.

BENEVIDES, S.D.; RAMOS, A.M.; STRINGHETA, P.C.; CASTRO, V.C. Qualidade da manga e polpa de manga Ubá. Ciência e Tecnologia de Alimentos, Campinas, v. 28, n. 3, p. 571-578, 2008.

BRASIL. Ministério da Agricultura e do Abastecimento. Instrução Normativa n 01 , de 7 de janeiro de 2000. Diário Oficial da União. Brasília, 10 de janeiro de 2000. Anexo II.

CANUTO, G.A.B.; XAVIER, A.A.O.; NEVES, L.C.; BENASSI, M.T. Caracterização físico-química de polpas de frutos da Amazônia e sua correlação com a atividade antirradical livre. Revista Brasileira de Fruticultura, Jaboticabal, v.32, n.4, p. 1198-1205, 2010.

CARVALHO, C. R. L. et al. Avaliação de cultivares de mangueira selecionadas pelo Instituto Agronômico comparadas a outras de importância comercial. Revista Brasileira de Fruticultura, Jaboticabal-SP, v. 26, n. 2, p. 264-271, Ago. 2004. 
CAVALCANTE, I.H.L.; MARTINS, A.B.G. Physical and chemical characterization of dovyalis fruits. International Journal of Fruit Science, New York, v.5, n.4, p.39-46, 2005.

DONADIO, C. D.; NACHTGAL, J.C.; SACRAMENTO, C. K. Frutas exóticas. Jaboticabal: FUNEP, 1998. 279p.

DONADIO, L.C.; MORO, F.V.; SERVIDONE, A.A. Frutas brasileiras. Jaboticabal: Novos Talentos, 2002. 288p.

FIGUEIREDO, J.O. Variedades comerciais. In: RODRIGUEZ, O.; VIÉGAS, F.; POMPEU JR., J.; AMARO, A.A. Citricultura brasileira. 2.ed. Campinas: Fundação Cargill, 1991. v.1, p.228-257.

LOPES, A. L.; MATTIETTO, R. A.; MENEZES, H. C. Estabilidade da polpa de pitanga sob congelamento. Ciência e Tecnologia de Alimentos, Campinas, v. 25, n.3, p. 553-559, 2005.
PIO, R.M.; FIGUEIREDO, J. O.; STUCHI, E.S.; CARDOSO, S.A.B. Variedades copas. In: MATTOS JR., D.; NEGRI, J.D.; PIO, R.M.; POMPEU JR., J. Citros. Campinas: Instituto Agronômico e Fundag, 2005. cap. 3, p. 37-60

REDD, J.B.; HENDRIX JÚNIOR, C.M.; HENDRIX, D.L. Quality control manual for citrus processing plants. Florida: Intercit, 1986. 250p.

VIÉGAS, F. C. P. A industrialização dos produtos cítricos. In: RODRIGUEZ, O. et al. (Ed.). Citricultura brasileira. 2. ed. Campinas: Fundação Cargill, 1991. p. 898-922. 